\title{
Deteç̧ão do Rearranjo da Proteína BCL2/JH em Carcinomas Epidermoides de Boca e Faringe
}

\section{Detection of Protein BCL2/JH Rearrangement in Epidermoid Carcinomas of Mouth and Pharynx}

\author{
Jair Montovani*, Magaly M. Sales**, Maria Inês M. C.Pardini***.
}

\author{
* Livre-Docente em Otorrinolaringologia. Professor Livre-Docente, Adjunto. \\ ** Doutorado e Pós-Doutorado em Genética pela UNESP. \\ **** Doutorado. Professora Doutora do Departamento de Clínica Médica, Divisão Hemocentro. \\ Instituição: Faculdade de Medicina de Botucatu - UNESP Departamento de Oftalmologia, Otorrinolaringologia e Cirurgia de Cabeça e Pescoço. \\ Botucatu / SP - Brasil. \\ Endereco para correspondência: Jair Cortez Montovani - Faculdade de Medicina de Botucatu - UNESP Departamento de Oftalmologia, Otorrinolaringologia e Cirurgia \\ de Cabeça e Pescoço Distrito de Rubião Júnior s/n - Botucatu / SP - Brasil - CEP: 18603-970 - Telefone/Fax: (+55 14) 3811-6256/ $3811-6081$ - E-mail: \\ montovan@fmb.unesp.br \\ Artigo recebido em 16 de Dezembro de 2009. Artigo aprovado em 15 de Maio de 2010.
}

\section{RESUMO}

Introdução:

A proteína BCL2 encontrada na membrana mitocondrial interna, regula a apoptose inibindo a morte celular programada. A translocação (14;18), detectada em 70 a 85\% dos linfomas foliculares, leva a superexpressão da proteína BCL2, pela justaposição do gene BCL2 ao segmento JH do gene da cadeia pesada da imunoglobulina. Porém, os achados da expressão da BCL2 em carcinoma de cabeça e pescoço são contraditórios.

Objetivo: Investigar a presença da translocação $(14 ; 18)$ do gene BCL2 em carcinomas de cabeça e pescoço.

Método: Foram examinadas 16 amostras de DNA, sendo 13 de carcinomas de células escamosas (CCE) e 3 de epidermoide (CE), por meio da reação em cadeia da polimerase (PCR).

Resultados: O rearranjo BCL2/JH foi encontrado em 2 (15\%) dos 13 casos de CCE e em nenhum dos 3 casos de CE. A média de frequência de moléculas com rearranjo foi de 46,44 x 107. Não foi observada associação entre a presença de rearranjo e a exposição ao tabaco e álcool ( $p=0,6545)$.

Conclusão: Diferente dos resultados encontrados em linfomas foliculares a presença da translocação $(14 ; 18)$ em carcinomas de cabeça e pescoço não é comum e, quando ocorre, pode ser uma mutação ocasional não associada a exposição ao tabaco e álcool.

Palavras-chave: translocação genética, biologia molecular, neoplasias bucais, neoplasias faríngeas.

\section{SUMMARY}

Introduction:

The BCL2 protein found in the internal mothocondrial membrana regulates the apoptosis preventing the programmed cell death. The translocation (14:18), detected in 70 to $85 \%$ of the follicular lymphoma, lead the super expression of BCL2 protein, by juxtaposition of BCL2 gene to the JH segment of the immunoglobulins' heavy chain gene. However, the found of the BCL2 expression in head and neck carcinoma are contradictious.

Objective:

Method:

To investigate the presence of the translocation (14:18) of the BCL2 gene in head and neck carcinoma. Sixteen DNA samplers were examinated being 13 of squamous cells carcinoma (SCC) and 3 of epidermoid (CE), y means of chain reaction of the polymerase (PCR).

Results:

Conclusion: The BCL2/JH rearrangement in $2(15 \%)$ of the CCE 13 cases and in none of the 3 cases of CE. The average of the frequency of molecules with rearrangement was $46,44 \times 107$. Was not observed association between the rearrangement presence and the exhibition to tobacco and alcohol $(p=0,6545)$.

Different from the results found in follicular lymphoma, the presence of the translocation $(14 ; 18)$ in head and neck carcinomas is not common and, when it occurs, it can be an occasional mutation not associated to exhibition to the tobacco and alcohol.

Keywords: genetics translocation, molecular biology, buccal neoplasia, pharyngal neoplasia. 


\section{INTRODUÇÃO}

O gene humano BCL2 está localizado no cromossomo 18q21 numa orientação telômero-centrômero e consiste de três exons (Figura 1). A proteína BCL2 encontrada na membrana mitocondrial interna, regula a apoptose, inibindo as células da morte celular programada (1). A translocação $(14 ; 18)$ (q32;q21), detectada em 70-85\% dos linfomas foliculares, leva a superexpressão da proteína BCL2 pela justaposição do gene BCL2 aos segmentos JH do gene da cadeia pesada da imunoglobulina (IGH). A maioria dos pontos de quebras da $t(14 ; 18)$ ocorre na região não codificadora 18q21.3 do BCL2. Estes pontos de quebras constituem as regiões MBR (Major Breakpoint Region), encontrada em aproximadamente $60 \%$ dos linfomas com a t(14;18) e mcr (Minor Cluster Region), localizada a $20 \mathrm{~kb}$ de distância da região $\operatorname{MBR}(2,3,4,5)$.

Dados de estudos mais recentes são contraditórios na correlação entre a presença de alterações dos rearranjos e expressão do gene BCL2/JH como valores preditivos de prognóstico dos linfomas foliculares (RADOJKOVIE et al., 2008) (6). Entretanto, para Deghied et al. (2007) (7) a expressão dessa proteína pode ser usada, inclusive restrospectivamente, para o diagnóstico de casos duvidosos, como por exemplo, hiperplasias reativas. Já em pacientes com expressões mínimas de BCL2/JH em amostras de sangue periférico ou da medula óssea o correto seria testar novamente o rearranjo de BCL2/JH para excluir falsos positivos $(8,9)$.

Diferente dos achados em linfomas, a superexpressão da proteína BCL2 tem sido detectada em 30\% dos carcinomas epidermoides de cabeça e pescoço $(10,11,12)$, não estando associada a presença da translocação t $(14 ; 18)$ (Harn et al., 1996). Poucos estudos epidemiológicos têm indicado que o uso de tabaco e o consumo elevado de álcool são fatores etiológicos importantes na indução de alterações gênicas como a translocação cromossomial t $(13,14,21)$. Porém, o rearranjo BCL2/JH tem sido encontrado em linfócitos de indivíduos sadios fumantes $(14,15,16,17,18)$ e em células da medula óssea de pacientes com doenças não linfoides $(8,17)$.

O objetivo deste estudo foi investigar a presença do rearranjo BCL2(MBR)/JH em amostras teciduais de pacientes com carcinomas de boca e faringe e a possível correlação do mesmo com a exposição ao tabaco e álcool.

\section{MÉTODO}

O DNA genômico foi extraído de 16 biópsias frescas de neoplasias de boca $(n=13)$ e faringe $(n=3)$

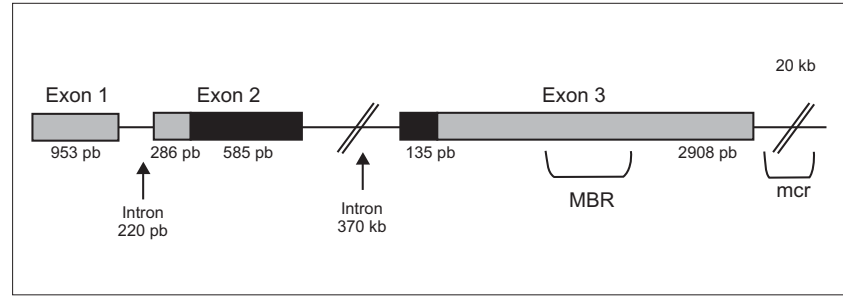

Figura 1. Estrutura do gene BCL2. O BCL2 contém três exons e dois introns. As regiões não traduzidas do gene estão ilustradas com as barras sólidas de cor cinza, enquanto que as barras de cor preta representam as regiões traduzidas.

obtidas de pacientes com histologia confirmada de carcinoma epidermoide, virgens de quaisquer tratamentos, através da digestão do tecido tumoral pela proteinase-K $(20 \mathrm{mg} / \mathrm{ml})$ e extração de DNA sem fenol-clorofórmio. As biópsias tumorais foram feitas no serviço Otorrinolaringologia da Faculdade de Medicina da UNESP, Botucatu, SP, Brasil, após os pacientes e ou responsáveis legais tomarem ciência dos objetivos da pesquisa e assinarem termo de consentimento livre e esclarecido e aprovado pelo Comitê de Ética em Pesquisa em seres humanos de nossa instituição.

A presença do rearranjo BCL2/JH (Figura 1), região de quebra MBR, foi investigada através do nested PCR. $1,0 \mu \mathrm{g}$ de DNA de cada amostra foi amplificado com 200 nmol dos primers MBR e JH (GribBen et al.,1994) em $50 \mu \mathrm{l}$ de tampão (Tris-HCl pH 7,5 a $5 \mathrm{mM}, \mathrm{KCl}$ a $25 \mathrm{mM} \mathrm{e}$ $\mathrm{MgCl} 2$ a 1,5 mM), 0,1 mM dNTPs e 1,25 U Taq DNA polimerase. Seguiram-se 25 ciclos de amplificação a $94^{\circ} \mathrm{C}$ por 1 minuto, $55^{\circ} \mathrm{C}$ por 1 minuto e $72^{\circ} \mathrm{C}$ por 1 minuto. Na segunda reação (nested) $10 \mu \mathrm{l}$ do produto da primeira reação foi reamplificado com os primers MBR-N e JH-N (200 nmol) em $50 \mu l$ de tampão (Tris- $H C l$ pH 7,5 a 8 mM, $\mathrm{KCl}$ a $40 \mathrm{mM}$ e $\mathrm{MgCl} 2$ a 1,5 mM), 0,1 mM de dNTPs e 1,25 U Taq DNA polimerase. Seguiram-se 30 ciclos de amplificação a $94^{\circ} \mathrm{C}$ por 1 minuto, $58^{\circ} \mathrm{C}$ por 1 minuto e, $72^{\circ} \mathrm{C}$ por 1 minuto. Os produtos do nested $\mathrm{PCR}$ foram submetidos à eletroforese em gel de poliacrilamida a $5 \%$ e corados em solução com brometo de etídeo $(50 \mathrm{mg} / \mathrm{ml})$.

DNA extraído da linhagem celular RL com o rearranjo MBR, foi utilizado como controle positivo da reação de PCR. DNA humano, negativo para a t(14;18) e, um tubo de PCR sem DNA foram utilizados como controles negativos da reação.

O teste exato de Fisher foi usado para avaliar a correlação entre a presença do rearranjo $\mathrm{BCl} 2(\mathrm{MBR}) / \mathrm{JH}$ e a exposição ao tabaco e álcool. 
Tabela I. Incidência dos casos de carcinoma epidermoide de boca e faringe.

\begin{tabular}{|c|c|c|c|c|c|c|c|}
\hline \multirow[b]{2}{*}{ Paciente } & \multirow[b]{2}{*}{ Idade/Sexo } & \multicolumn{2}{|c|}{ Exposição } & \multirow[b]{2}{*}{ Sítio } & \multirow[b]{2}{*}{ Histologia } & \multirow[b]{2}{*}{ Grau } & \multirow[b]{2}{*}{$\mathrm{BCL} 2 / \mathrm{JH}$} \\
\hline & & Tabaco & Álcool & & & & \\
\hline | & $43 / M$ & $\bullet$ & $\bullet$ & Assoalho da boca & CE & $B$ & - \\
\hline 2 & $44 / M$ & $\bullet$ & $\bullet$ & Assoalho da boca & CE & $B$ & + \\
\hline 3 & $49 / M$ & $\bullet$ & $\bullet$ & Assoalho da boca & CE & B & - \\
\hline 4 & $52 / M$ & $\bullet$ & $\bullet$ & Orofaringe & CE & $M$ & - \\
\hline 5 & $53 / M$ & $\bullet$ & $\circ$ & Palato & CE & $M$ & - \\
\hline 6 & $56 / M$ & $\bullet$ & $\bullet$ & Palato & CE & B & + \\
\hline 7 & $60 / M$ & $\bullet$ & $\bullet$ & Palato & CE & $M$ & - \\
\hline 8 & $63 / M$ & - & $\bullet$ & Mucosa retromolar & CE & $M$ & - \\
\hline 9 & $64 / M$ & $\circ$ & $\circ$ & Mucosa bucal & CE & B & - \\
\hline 10 & $65 / M$ & $\bullet$ & $\bullet$ & Palato & CE & $M$ & - \\
\hline | | & $67 / M$ & $\circ$ & $\circ$ & Palato & CE & $M$ & - \\
\hline 12 & $69 / M$ & $\bullet$ & $\circ$ & Mucosa bucal & CE & B & - \\
\hline 13 & $72 / F$ & $\circ$ & $\circ$ & Mucosabucal & CE & B & - \\
\hline 14 & $72 / M$ & - & $\bullet$ & Língua & CE & B & - \\
\hline 15 & $80 / M$ & $\circ$ & $\circ$ & Língua & CE & B & - \\
\hline 16 & $83 / F$ & $\circ$ & $\circ$ & Língua & CE & B & - \\
\hline
\end{tabular}

Legenda: CE: carcinoma epidermoide; M: masculino; F: feminino; B: bem diferenciado; MD: moderadamente diferenciado; (-): expressão negativa; (+): expressão positiva.

Tabela 2. Associação entre a presença do rearranjo MBR e a exposição ao tabaco e álcool em pacientes com tumores de cabeça e pescoço.

\begin{tabular}{|c|c|c|c|c|}
\hline \multirow{2}{*}{$\begin{array}{l}\text { Presença do rearranjo } \\
\mathrm{BCL} 2 / J \mathrm{H}\end{array}$} & \multirow{2}{*}{$\begin{array}{l}\text { Número } \\
\text { de casos }\end{array}$} & \multirow{2}{*}{$\begin{array}{l}\text { Não Exposição } \\
\text { Tabaco e Álcool }\end{array}$} & \multicolumn{2}{|c|}{ Exposição } \\
\hline & & & Tabaco e Álcool & Tabaco \\
\hline Positivo & 2 & 0 & 2 & 0 \\
\hline Negativo & 14 & 5 & 7 & 2 \\
\hline Total & 16 & 5 & 9 & 2 \\
\hline
\end{tabular}

Legenda: $p=0,6545$ (Teste exato de Fisher).

\section{RESULTADOS}

A incidência do rearranjo BCL2(MBR)/JH foi de $12,5 \%$ (2/16) para os casos de carcinoma epidermoide de boca e faringe (Tabela 1 ).

A análise da frequência do rearranjo BCL2(MBR)/JH foi feita por molécula $(\lambda)$ nos dois pacientes positivos (Figuras 2, 3 e 4) e usou-se um modelo Poisson de análise estatística para o número de rearranjos por amostra (21). Como todas as triagens apresentavam o mesmo número de moléculas ( $1 \mu \mathrm{g}$ de DNA contém 5 x 10-19 moles de cada cópia de sequência única $=\sim 300.000$ moléculas), a estimativa $\lambda$ foi dada pelo estimador Poisson usual: $\lambda=1 / M \cdot \ln (1-p)$, onde $p$ é a fração de triagens com pelo menos um rearranjo.

Nenhuma associação foi encontrada entre a presença do rearranjo BCL2(MBR)/JH e a exposição ao tabaco e álcool (Tabela 2) $(\mathrm{P}=0,6545)$.
Os produtos de PCR dos pacientes positivos para o rearranjo BCL2/JH, podem ser observados na Figura 2. Fragmentos de tamanhos diferentes foram observados entre os dois pacientes com carcinoma de célula escamosa.

As Figuras 2, 3 e 4 mostram as amplificações, realizadas em triagens múltiplas, do rearranjo BCL2(MBR)/ $\mathrm{JH}$ dos dois pacientes que foram positivos.

O paciente 2, tabagista desde criança e etilista crônico ( dois litros de álcool por dia), permaneceu positivo em 3 de mais 8 triagens realizadas (Figura 2A). Foram observados fragmentos de mesmo tamanho, sugerindo tratar-se do mesmo clone. A frequência do rearranjo MBR foi de 19,32 x 10-7 moléculas positivas.

O paciente 6, tabagista e etilista crônico, permaneceu positivo em 7 de mais 8 triagens realizadas (Figura 2B). Foram observados fragmentos de mesmo tamanhos ( 200 


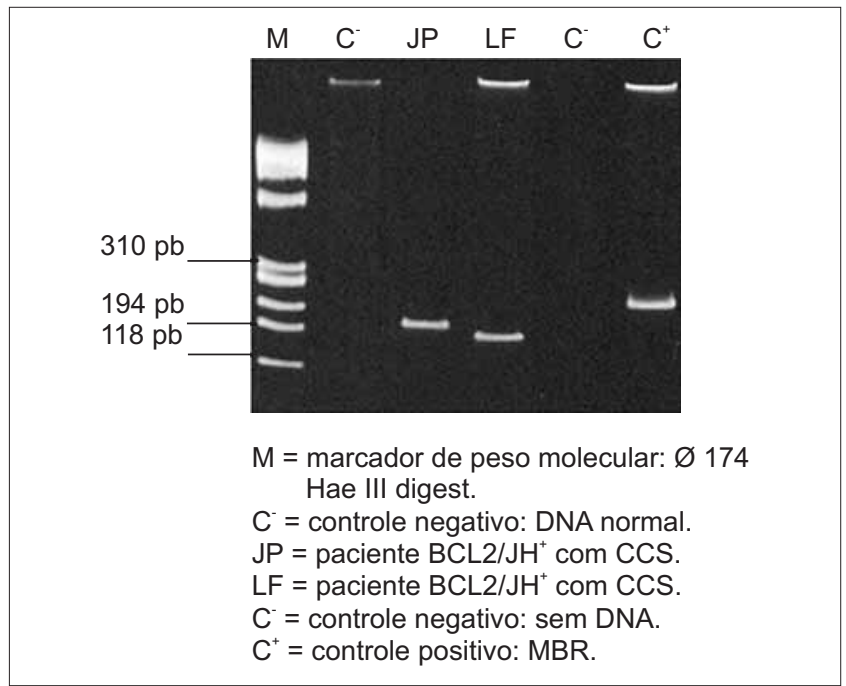

Figura 2. Amplificações do rearranjo BCL2(MBR)/JH em pacientes com carcinoma epidermoide de boca (CE). Eletroforese em gel de poliacrilamida 5\%.

pb). A frequência do rearranjo MBR foi de $73,57 \times 10^{-7}$ moléculas positivas.

\section{DIsCUSSÃO}

Uma expressão elevada do BCL2 tem sido observada em carcinomas de células escamosas (LAvieille et al., 1998; DrenNing et al., 1998), porém, não existem relatos na literatura da presença do rearranjo BCL2/JH em tumores de cabeça e pescoço. No presente estudo, o rearranjo BCL2(MBR)/JH foi investigado em biópsias frescas de 13 carcinomas de células escamosas e de 3 carcinomas epidermoides, provenientes de pacientes com tumores de cabeça e pescoço, pelo nested PCR.

HARN et al. (1996) (12) relataram a ausência do rearranjo MBR ao analisarem 32 casos de carcinoma de nasofaringe através da técnica de PCR. Entretanto, no estudo de Harn et al. (1996) (12) foram utilizadas amostras de tecido fixado. Assim, esta discordância na incidência do rearranjo BCL2/JH pode ser devido a diferenças técnicas ou amostrais.

SALo et al. (1997) (20) estudaram o estado do gene BCL2 em 9 linhagens celulares humanas de carcinoma de células escamosas. O cDNA do BCL2 foi amplificado em 5 das linhagens celulares, mostrando que o mRNA estava expresso nestas células. Os 5 cDNAs foram sequenciados, porém, mutações de ponto no gene BCL2 não foram detectadas, indicando que a translocação é provavelmente uma mudança típica nestas células neoplásicas. Para Vergier et al. (2004) (18) esses resultados sugerem que um dos mecanismos da superexpressão do BCL2 em carcinomas

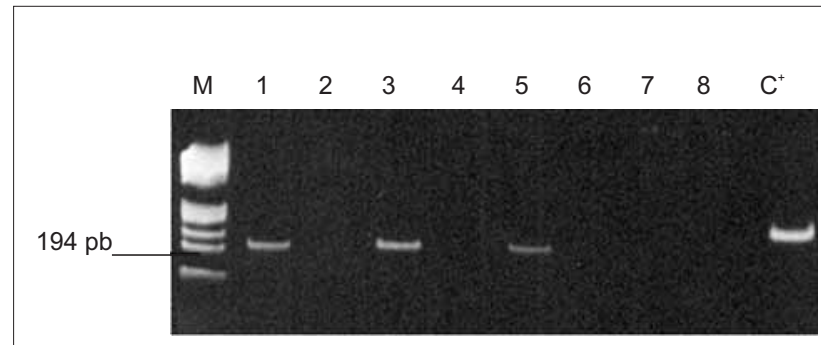

$M=$ marcador de peso molecular: $\varnothing 174$ Hae III digest. 1 a 8) Triagens do paciente 2 que foi positivo para 0 rearranjo MBR: poços 1, 3, 5 no gel.

$\mathrm{C}^{+}=$Controle positivo: $\mathrm{MBR}$.

Figura 2A . Amplificações múltiplas do rearranjo BCL2(MBR)/ $\mathrm{JH}$ em paciente com carcinoma epidermoide de boca (CE). Eletroforese em gel de poliacrilamida $5 \%$.

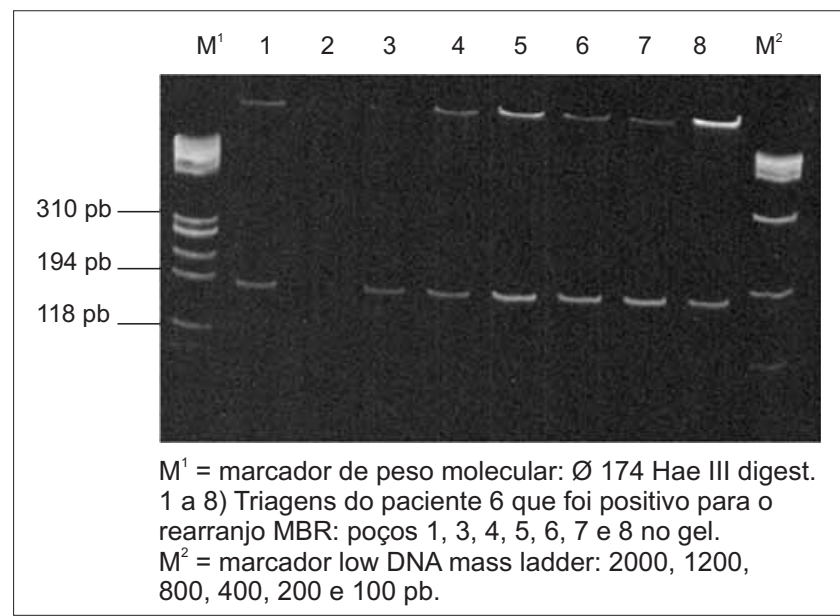

Figura 2B. Amplificações múltiplas do rearranjo BCL2(MBR)/ $\mathrm{JH}$ em paciente com carcinoma epidermoide de boca (CE). Eletroforese em gel de poliacrilamida 5\%.

de célula espinocelulares, pode ser devido a translocação cromossômica t( $14 ; 18)$.

Bell et al. (1995) (13) e SHuler et al. (2003) (17) relataram uma associação significativa entre o hábito de fumar e a frequência de células com a t(14;18) no sangue periférico de voluntários sadios. Outros autores encontraram essas mesmas translocações em indivíduos com câncer associado são tabaco $(8,9,15,16)$. No presente estudo, não foi observada uma associação entre a positividade do rearranjo MBR/BCL2 e a exposição ao tabaco e álcool nos pacientes com tumores de cabeça e pescoço. Como a taxa da mutação BCL2 foi idêntica tanto para fumantes e não fumantes $(8,9,15)$, a relação antígenos do tabaco estimu- 
lando o aparecimento de clones raros de rearranjos BCL2/ JH pré-existentes não foi observado nos nossos pacientes. Entretanto, a ausência dessa associação ficou prejudicada pelo baixo número de indivíduos estudados.

Mesmo assim, no presente estudo, um dos pacientes fumantes positivos apresentou uma frequência elevada $\left(73,57 \times 10^{-7}\right)$ do rearranjo BCL2 (MBR)JH e esse aumento pode ser devido à expansão dominante de um único clone e o sequenciamento do produto de PCR desse indivíduo pode confirmar essa observação. Esse dado poderia ter valor preditivo de diagnóstico como o mostrado por Bell et al. (1995), quando um dos indivíduos fumantes (três maços de cigarros por dia), que apresentou uma frequência elevada do rearranjo BCL2/JH no sangue periférico, desenvolveu melanoma (21). Este indivíduo havia sido positivo 4 vezes em 4 triagens realizadas e permaneceu positivo em mais 2 de 4 triagens retestadas 8 meses mais tarde. Assim, a mensuração da taxa de translocações $\mathrm{t}(14 ; 18)$ poderia identificar indivíduos com um risco maior de desenvolvimento de linfoma ou outros cânceres ou então que responderam mal a terapia $(6,7,21)$.

Enfim, pode-se dizer que o rearranjo BCL2(MBR)JH não está restrito a doenças linfoproliferativas e pode ser detectado em células neoplásicas de carcinomas primários de boca e faringe. A translocação t $(14 ; 18)$, portanto, pode ser uma mutação secundária encontrada em carcinoma espinocelulares de boca e faringe mas em nosso caso, devido ao pequeno número de pacientes testados, não se pôde avaliar o valor prognóstico da presença do rearranjo BCL2/JH nestes tumores.

\section{REFERÊNCIAS BIBLIOGRÁFICAS}

1. Eckenrode EF, Yang J, Velmurugan GV, Foskett JK, White C. Apoptosis protection by $\mathrm{Mcl}-1$ and $\mathrm{Bcl}-2$ modulation of inositol 1,4,5-Trisphosphate receptordependent $\mathrm{Ca}^{2+}$ signaling. J. Bid. Chem. 2010, 285:285-97.

2. Lee MS. Molecular aspects of chromosomal translocation t(14;18). Seminars in Hematology. 1993, 30:297-305.

3. Heim S, Miltelman F. Malignant Lymphomas. (1995). In: Cancer Cytogenetics (Heim, S. and Miltelman, F., eds.). New York: Wiley-Liss, pp. 266-309.

4. Gaidano G, Dalla-Favera R. (1997). Molecular Biology of lymphomas. In: Cancer: Principles and Practice of Oncology (Devita Jr, V.T., Hellman, S. and Rosenberg, S., eds.). Philadelphia: J. B. Lippincott Company, pp.21312145.

5. Monni O, Franssila K, Joensuu H, Knuutila S. BCL2 overexpression in diffuse large B-cell lymphoma. Leukemia and Lymphoma. 1999, 34:45-52.

6. Radojkovic M, Ristic S, Colovic M, Cemerikic-Martinovic V, Radojkovic D, Krtilica K. Molecular characteristics and prognostic significance of $\mathrm{Bcl}-2 / \mathrm{IgH}$ gene rearrangement in Serbian follicular lumphoma patients. Neoplasma. 2008, 55(5):421-7.

7. Deghedy H, Fonda M, Sharin D, Shamaa S, El-Bedewy A, Ghaffar HA. Diagnostic and prognostic utility of t $(14 ; 18)$ in follicular lymphoma. Acta Haematologica. 2007, 118(4):231-6.

8. Bownan A, Jones D, Medeiros J, Luthra R. Quantitative PCR detection of $\mathrm{t}(14 ; 18) \mathrm{Bcl} 2 / \mathrm{JH}$. Fusion sequences in follicular lymphoma patients. JMD. 2004, 6(4):129-36.

9. Karubec K, Guo Y, Suzumiya J et al. CD10(-) MUM1(+) follicular lymphomas lacks BCL2 translocation and clinical features. Blood. 2007, 109:3076-9.

10. Lavieille JP, Gazzeri S, Riva C, Reyt E, Brambilla C, Brambilla E. p53 mutations and p53, Walf-1, Bax and Bcl2 expression in field cancerization of the head and neck. t(14;18). Anticancer Res. 1998, 18:4741-9.

11. Drenning SD, Marcovitch AJ, Johnson DE, Melhem MF, Tweardy DJ, Grandis JR. Bcl-2 but not Bax expression is associated with apoptosis in normal and transformed squamous epithelium. Clin Cancer Res. 1998, 11:291321.

12. Harn HJ, Ho LI, Liu CA, Liu GC, Lin FG, Lin JJ, Chang JY, Lee WH. Down regulation of bcl-2 by p53 in nasopharyngeal carcinoma and lack of detection of its specific t $(14 ; 18)$ chromosomal translocation in fixed tissues. Histopathology. 1996, 28:317-23.

13. Bell DA, Liu Y, Cortopassi GA.Occurrence of bcl-2 oncogene translocation with increased frequency in the peripheral blood of heavy smokers. J Natl Cancer Inst. $1995,87: 223-4$

14. Hirose Y, Masaki Y, Ozaki M. Fluorescence in situ hybridization of chromossome FgH/BCL2 trabslocations for paraffin-embedded tissue: evaluation in follicular lymphomas. Int J Hematol. 2003, 78:154-9.

15. Schantz SP, Harrison LB, Forastiere AA. (1997). Tumors of the nasal cavity and paranasal sinuses, nasopharynnx, oral cavity, and oropharynx. In: Cancer: Principles and Practice of Oncology (Devita Jr, V.T., Hellman, S. and Rosenberg, S., eds.). Philadelphia:J. B. Lippincott Company, pp.741-801. 
16. Xu J, Gimenez-Conti IB, Cunnigham JE, Collet AM, Luna MA, Lanfranchi HE, Spitz MR, Conti CJ. Alterations of p53, cyclin D1, Rb, and H-ras in human oral carcinomas related to tobacco use. Cancer. 1998, 83:204-12.

17. Schuler F, Hirt C, Dolken G. Chromossomal translocation $\mathrm{t}(14 ; 18)$ in health individuals. Semim Cancer Biol. 2003, 13:203-9.

18. Vergier B, Beland-Rotureau MA, Benany MN, BeylotBarry M, Dubus P, Delaunay M, Garroste JC, Taine L, Merlio JP. Neoplastic cells do not carry bcl2-JH rearrangements detected in a sulset of primary cutaneous follicle center Bcell lymphomas. Am J Pathol. 2004, 28(6):748-5.

19. Rauzy O, Galoin S, Chale JJ, Adoue D, Albarede JL,
Delsol G, Saati TAL. Detection of $\mathrm{t}(14 ; 18)$ carrying cells in bone marrow and peripheral blood from patients affected by non-lymphoid diseases. J Clin Pathol: Mol Pathol. 1998, 51:333-8.

20. Salo A, Servomaa K, Kiuru A, Pulkkinen J, Grénman R, Pekkola-Heino K, Rytomaa T. The bcl-2 gene status of human head and neck cancer cell lines. Acta Otolarynngol. 1997, 529:233-6.

21. Fuscoe JC, Setzer RW, Collard DD, Moore MM. Quantification of $t(14 ; 18)$ in the lymphocytes of healthy adult humans as a possible biomarker for environmental exposures to carcinogens. Carcinogenesis. 1996, 17:1013-20. 